INTERACTION OF ALKALI ALKYL SULPHATES, ETC.

2371

\title{
CCXXI.-Interaction of Alkali Alkyl Sulphates and Alkali Nitrites: Theories of the Formation of Aliphatic Nitro-compounds.
}

By Panchānon Neogr.

RÂY and Neogi (T., 1906, 89, 1900) have shown that nitroethane and ethyl nitrite are both produced by the interaction of alkali nitrites and alkali salts of ethylsulphuric acid. On account of the theoretical importance of the general reaction of the formation of aliphatic nitro-compounds from alkali nitrites a systematic study of the reaction with respect to other alkylsulphuric acids has been undertaken. The results show that the reaction is quite a general one, and this reopens the general question of the mechanism of the formation of aliphatic nitrites and nitro-compounds. Until lately it was known that aliphatic nitro-compounds are only produced by the interaction of silver nitrite and alkyl iodides. Rây and Neogi (P., 1907, 23, 246), however, have shown that nitrites and nitrocompounds are both produced by the interaction of alkyl iodides and mercurous nitrite. It is now shown that nitro-compounds may be prepared by the action of alkali nitrites as well.

The formation of aliphatic nitrites and nitro-compounds is thus analogcus to the formation of cyanides and isocyanides by the reaction of silver cyanide with alkyl iodide, and of alkali cyanides with the alkali salts of alkylsulphuric acids. In view of this analogy, the applicability of the theories advanced in the case of cyanides and isocyanides may be discussed with advantage in connexion with the formation of nitrites and nitro-compounds.

(a) Tautomerism.-Laar's idea of tautomerism seems to be scarcely applicable to nitrites and nitro-compounds. In the first place, it is indisputable that nitrous acid has the constitution $\mathrm{HO} \cdot \mathrm{NO}$ as aqueous solutions of nitrous acid with alcohols yield nitrites only and not nitro-compounds. It therefore follows that alkali nitrites and silver nitrite, formed by the action of the corresponding hydroxides on the acid, cannot possess both hydroxylic and nitro-constitutions, although Reynolds without assigning any particular reason is disposed to think that "isomeric metallic nitrites of both types exist which are also tautomeric in a marked degree" (T.. 1903, 83, 643). As regards silver nitrite, Divers (T., $1883,43,455 ; 1885,47,205)$ was of the opinion that it has a nitro-constitution, whilst Rây and Gañguli (P., 1905, 21, 278) attempted to show that there are two varieties of silver nitrite, one obtained by precipitation and the other by careful recrystal- 
lisation of the precipitated nitrite. The author has, however, found that both varieties yield the same proportion of aliphatic nitro-compounds when reacting with ethyl iodide, and agrees with Divers (P., 1905, 21, 281), who maintained that the difference between the two is merely a physical one. With respect to mercurous nitrite Rây and Sen (T., 1903, 83, 491) think, owing to the formation of mercuric oxide in the products of the decomposition by heat, that "the atoms in some of the molecules of the nitrite arrange themselves as $\mathrm{Hg} \cdot \mathrm{NO}_{2}$, the non-oxylic form, and in others assume the oxylic form $\mathrm{HgO} \cdot \mathrm{NO}$, scission taking place in each case at the indicated point." It is inconceivable, however, that such a heavy atom as that of mercury would oscillate between nitrogen and oxygen in the manner suggested by Rây and Sen. Moreover, the very idea of silver, mercurous, or alkali nitrites being tautomerised runs counter to Laar's hypothesis, which is based on the mobility of the light hydrogen atom, and Wade ('T., 1902, 81, 1607) well puts it in the case of silver cyanide when he says "its extension to the heavy silver atom is not justified dynamically; an atom cannot vibrate in a stable condition around a group of one-fourth of its mass."

A modified form of tautomeric explanation has been offered by Rây and Neogi (T., 1906, 89, 1905), who remark that "the more correct view would seem to be that it is only during the substitution of the atom of the metal by the alkyl radicle that a tautomeric change takes place." This argument sounds plausible, although in this case also the comparative immobility of heavy higher alkyl radicles has not been considered, and a still greater objection arises from the fact that when substitution takes place between the alkyl radicle and hydrogen in the interaction of alcohols and nitrous acid, no tautomeric change takes place, as only nitrites are formed, and no nitro-compound. It seems, therefore, that some explanation other than tautomeric change would be more suitable.

(b) Isomeric Conversion. - Following Gautier's hypothesis (Compt. rend., 1867, 65, 468) of the possibility of isomeric conversion of cyanides and isocyanides, the isomeric conversion of nitrites into nitro-compounds at a higher temperature may be advanced as a possible hypothesis. The nitrite, of course, is formed by direct interchange as the inorganic nitrites possess oxylic constitution, and, at a higher temperature, becomes partly converted into the nitro-compound. This hypothesis has the merit of simplicity, and as the conversion would take place at a higher temperature it also explains why in the case of nitrous acid only the nitrite is obtained. The reaction between silver or mercurous nitrite and alkyl iodides takes place at $100^{\circ}$, and that between 
alkali nitrites and alkyl sulphates at higher temperatures. Moreover, the reaction in all cases takes several hours for completion, during which time the nitrite may slowly be converted into the nitrocompound until an equilibrium is reached, although it is difficult to explain how methyl and ethyl nitrites, which are gaseous at the ordinary temperature, would be capable of such isomeric conversion. In any case, experimental evidence of the conversion of nitrites into nitro-compounds is lacking, and until such evidence is forthcoming it would be difficult to say definitely whether the hypothesis of isomeric conversion would explain the formation of nitrocompounds in all cases.

(c) Additive Hypothesis.--Nef (Annalen, 1892, 270, 329) introduced the idea of intermediate additive compounds in the case of cyanides and isocyanides, which has been accepted and modified by Wade (T., 1902, 81, 1596). The formation of nitro-compounds may also be readily explained on the additive hypothesis in the following manner, the nitrite being formed by direct interchange.

(i) Silver nitrite and alkyl iodides,

$$
\mathrm{AgO} \cdot \mathrm{N}: \mathrm{O}+\mathrm{EtI}=\mathrm{AgO} \cdot \mathrm{NEt} \cdot \mathrm{OI}=\mathrm{O}: \mathrm{NEt}: \mathrm{O}+\mathrm{AgI} \text {. }
$$

Mercurous nitrite behaves in a similar manner with ethyl iodide.

(ii) Alkali salts of alkylsulphuric acids and alkali nitrites:

$\mathrm{KO} \cdot \mathrm{N}: \mathrm{O}+\mathrm{EtKSO}_{4}=\mathrm{KO} \cdot \mathrm{NEt} \cdot \mathrm{O} \cdot \mathrm{KSO}_{4}=\mathrm{O}: \mathrm{NEt}: \mathrm{O}+\mathrm{K}_{2} \mathrm{SO}_{4}$.

The $\cdot \mathrm{N}: \mathrm{O}$ group behaves in nitrites as $: \mathrm{N} \cdot \mathrm{O} \cdot$, the alkyl group attaching itself to one of the free bonds of the nitrogen atom, and the rest of the molecule attaching itself to the oxygen. Silver and iodine, mercury and iodine, and potassium and the group $\mathbf{K S O}_{4}$ being contiguous to each other naturally combine with each other and separate out. Experimental evidence, however, in this case also is lacking, as no such intermediate compounds have been actually isolated.

\section{ExperimentaL.}

With regard to the reaction between alkali nitrites and alkali salts of alkylsulphuric acids the following general facts should be noted in connexion with the experimental results:

(1) The reaction takes place only during fusion, there being no reaction if fusion does not occur. During fusion much alcohol is produced, and the reaction may be considered to take place in the presence of the corresponding alcoholic medium.

(2) A few drops of water, when added, help the reaction by lowering the temperature at which the reaction begins, although the yield is smaller. The reaction evidently takes place in some liquid menstruum, and seems to be ionic in nature.

(3) Nitric oxide is given off in all the reactions, and is due to the 
action of free sulphuric acid, obtained by the partial hydrolysis of the salts of alkylsulphuric acids, on the alkali nitrites. The potassium salt of amylsulphuric acid is the least stable of the potassium salts, and the yield of amyl nitrite is consequently the smallest, the yield of nitrite diminishing as the series is ascended.

The apparatus employed in the case of the salts of methylsulphuric acid* was slightly different from what was used in the case of the salts of ethylsulphuric acid (Rây and Neogi, T., 1906, $89,1900)$. The mixture was contained in a round-bottomed flask with a short neck (instead of a glass retort), provided with an

\section{[With Birendra Bhushan Adhicary.]}

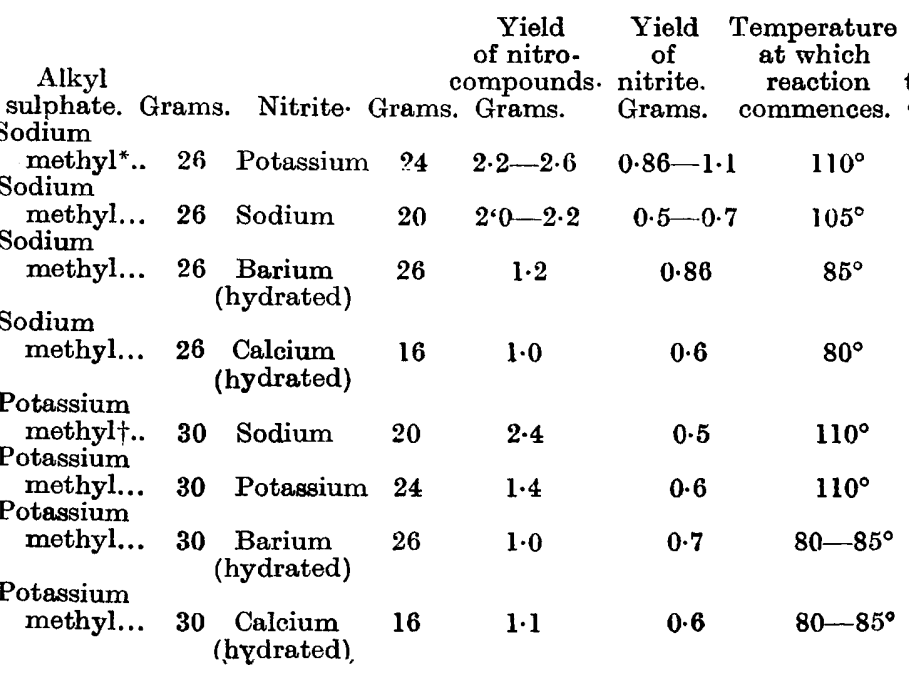

* When the apparatus was disconnected a strong odour of methylamine was observed in the issuing gas which iustantly turned red litmus blue. The formation of methylamine was probably due to the partial reduction of nitromethane by the potassium nitrite, which was already present in excess. The gas also contained ammonia, which would be due to the decomposition of hydroxylamine formed by the reduction of methyl nitrite by means of potassium nitrite. The formation of ammoniacal compounds in the case of all the methyl compounds is remarkable, as the corresponding amines were not formed in the case of the higher members of the series. It is to be noted that Wade ('T., 1902, 81, 1596) also obtained the corresponding amines in the interaction of alkali cymides and the alkali salts of alkylsulphuric acids.

+ Yield of nitro-compound about 20 per cent. of the theoretical. No liquid of higher boiling point as in the case of the ethyl compound.

* Kaufler and Pomeranz (Monatsh., 1961, 22, 492) and Walden (Ber., 1907, 40, 3214) have prepared aliphatic nitro-compounds by the interaction of methyl sulphate and a solution of potassinm nitrite. 


\section{[With Tarini Charan Chowdhuri.]}

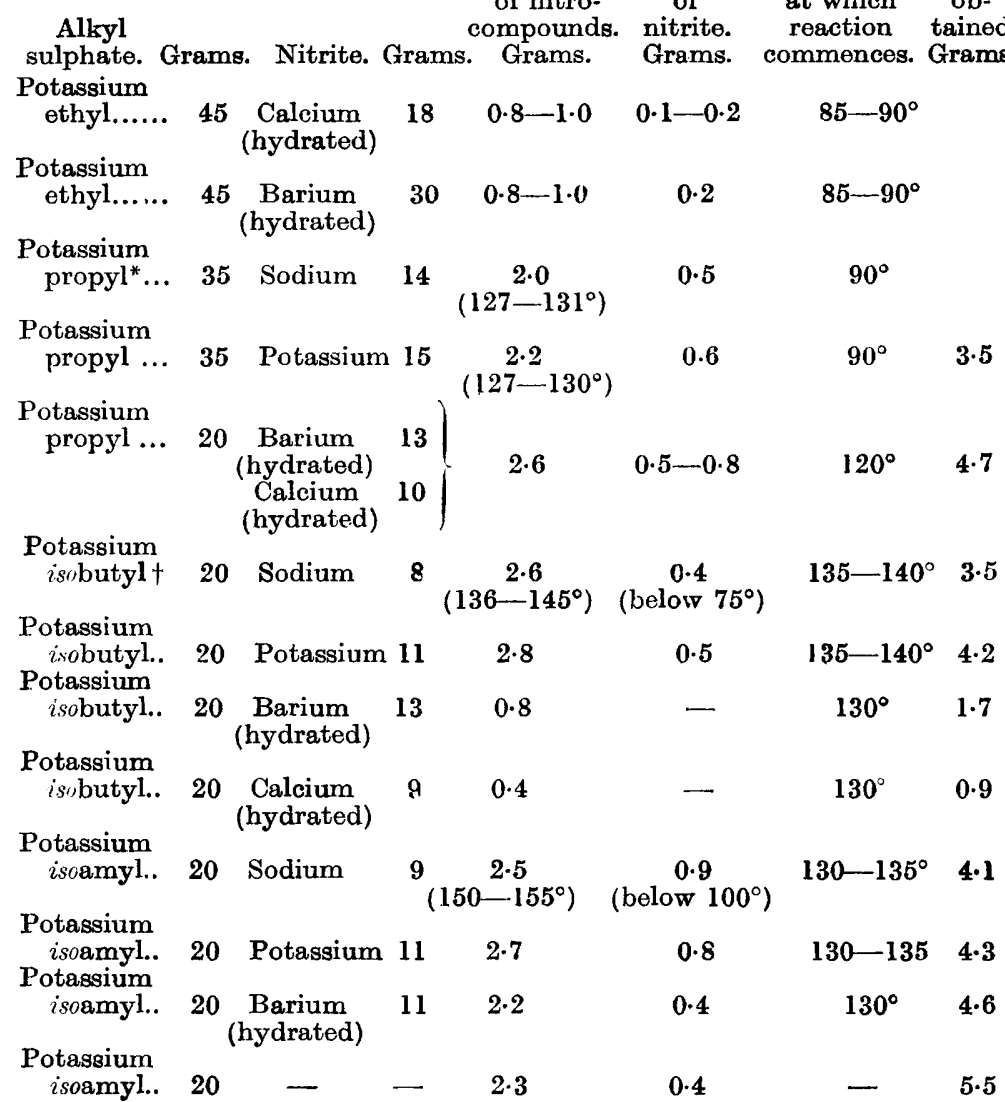

* The oil obtained after the distillation of the residue in the case of propyl, isobutyl, and isoumyl compounds consists of a mixture of the corresponding nitrite and nitro-compound, together with small quantities of the corresponding alcohol. It is difficult to separate them, and the weights given are only apnroximate.

$\dagger$ The oil obtained in the case of isobutyl, and especially amyl componnds, contains a good deal of the corresponding alcohol.

upright condenser. The flask was immersed in an oil-bath instead of a glycerol-bath. The reaction was conducted in a current of carbon dioxide, and methyl nitrite was absorbed in 90 per cent. alcohol contained in three or four Muencke's bottles immersed in ice-cold water. When the reaction was over, the liquid in the flask was distilled under diminished pressure as long as any oil passed 
over, and the oily layer was separated, washed, and dried. In the case of the salts of the higher sulphuric acids, the mixture was simply heated in an oil-bath in a flask with an upright condenser, and distillation was effected under the ordinary pressure up to $200^{\circ}$. The nitrite was estimated in alcoholic solution according to the method of the British Pharmacopoia, and nitro-compounds were further recognised by their characteristic reaction with alcoholic sodium hydroxide, when they solidified en masse owing to the formation of their sodium compounds.

Rajshahi Coltege, Rathihah, Bengal, india. 